06

\title{
Особенности пироэффекта в эпитаксиальных слоях нитрида алюминия, полученных на Si-подложках
}

\author{
(ㄷ Г.А. Гаврилов, К.Л. Муратиков, Е.А. Панютин \\ Физико-технический институт им. А.Ф. Иоффре РАН, Санкт-Петербург, Россия \\ `E-mail: eugeny.panyutin@mail.ioffe.ru
}

Поступило в Редакцию 16 сентября 2019г.

В окончательной редакции 30 сентября 2019 г.

Принято к публикации 2 октября 2019 г.

\begin{abstract}
Проведены исследования пироэффекта в квазиобъемных AlN-слоях с толщиной 10-170 $\mu$ m, полученных по технологии хлоридно-гидридной газофазной эпитаксии на стандартных Si-подложках. Измерение пироэлектрического тока осуществлялось методом теплового воздействия нестационарного (типа „меандр“) лазерного излучения, что в совокупности с данными независимого бесконтактного измерения динамики поверхностной температуры активного слоя позволило определить значение пирокоэффициента AlN в составе биморфной AlN/Si-структуры для различной толщины AlN-слоев. Установлено, что эти значения в среднем меньше тех, которые соответствуют материалу, полученному по той же технологии, но на $\mathrm{SiC}$ подложках, и для достижения значений пирокоэффициентов, сравнимых по величине, в случае „AlN на $\mathrm{Si}^{*}$ требуется увеличение толщины слоев $\mathrm{AlN}$ на 50-60\%. Вместе с тем при большой толщине AlN-cлоя $(110,170 \mu \mathrm{m})$ после удаления кремниевой подложки значения пирокоэффициента возрастали и достигали величины $\sim 8.6-9.0 \mu \mathrm{C} /\left(\mathrm{m}^{2} \cdot \mathrm{K}\right)$.
\end{abstract}

Ключевые слова: пироэффект, нитрид алюминия, пирометрические сенсоры, высокоинтенсивное лазерное излучение.

DOI: 10.21883/PJTF.2020.01.48858.18039

В настоящее время наиболее качественные эпитаксиальные слои широкозонных III-нитридов выращиваются на дорогостоящих сапфировых и карбидокремниевых подложках. В то же время экономическая целесообразность стимулирует поиски таких технологий, которые обеспечили бы для GaN или AlN переход - c минимальной потерей структурного совершенства на существенно более доступные кремниевые подложки [1-3]. Тем не менее проблему получения таких структур, в частности малодислокационных структур типа „AlN на $\mathrm{Si}^{\text {“6 }}$ необходимого приборного качества, пока можно признать решенной лишь частично, причем наибольшие сложности возникают при осаждении толстых $(>10 \mu \mathrm{m})$ и, в особенности, квазиобъемных слоев, получаемых методом хлорид-гидридной газофазной эпитаксии (HVPE-метод), обеспечивающим высокие скорости роста [4].

В связи с этим одна из актуальных задач современной твердотельной электроники состоит не только в оптимизации технологических процессов роста $\mathrm{AlN}$, но и в изучении его физических свойств в тех аспектах, где повышенная дефектность слоев не является особо критичной. Более того, исключительная термостабильность диэлектрических параметров этого материала и перспективы его практического применения [5,6] способствуют возрастанию интереса к изучению различных физических свойств $\mathrm{AlN}$, в том числе и пироэлектрического эффекта [7]. Хотя пироэффект уже был предметом изучения в некоторых более ранних работах [8-10], однако эпитаксиальные пленки для этих исследований были получены либо на $\mathrm{SiC}$ [9], либо на карбонизированном кремнии $(\mathrm{SiC} / \mathrm{Si})[10]$.

Целью настоящей работы является исследование пироэффекта в квазиобъемных эпитаксиальных слоях нитрида алюминия, полученных на стандартных промышленных $\mathrm{Si}(111)$-подложках, поверхность которых не была подвергнута какой-либо предварительной модификации, а также их сравнение со свойствами аналогичных слоев, выращенных хотя и на инородных, но все же более совместимых с AIN подложках.

HVPE-метод, использованный в работе для получения слоев AlN на Si-подложках, был во многом аналогичен технологии, описанной ранее [9] (горизонтальный проточный реактор, газ-носитель - аргон, реакционная смесь $\mathrm{NH}_{3}$ и $\mathrm{AlCl}_{3}$ в соотношении 3:5, температура $T=1050^{\circ} \mathrm{C}$ ), которая, однако, тогда применялась для

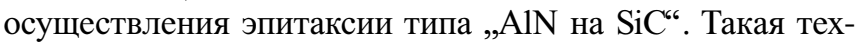
нология в некоторых режимах допускает весьма высокие скорости роста (для AlN до $25 \mu \mathrm{m} / \mathrm{h}$ ), что позволило получить набор образцов в широком диапазоне толщин эпитаксиального слоя. Структурное совершенство полученных таким способом слоев AlN на Si-подложках, оцениваемое в рамках рентгеноструктурного анализа по полуширине кривой качания ( 50-60 arcmin), несколько уступало „рентгеновскому“ качеству нитрида алюминия, рост которого осуществлялся в аналогичных условиях, но на подложках $6 H-\mathrm{SiC}$ и $4 H-\mathrm{SiC}(15-20 \mathrm{arcmin})$.

Было исследовано несколько образцов AlN/Si-структур с контактными электродами на основе серебряной пасты типа „Degussa“; площадь активного слоя совпа- 


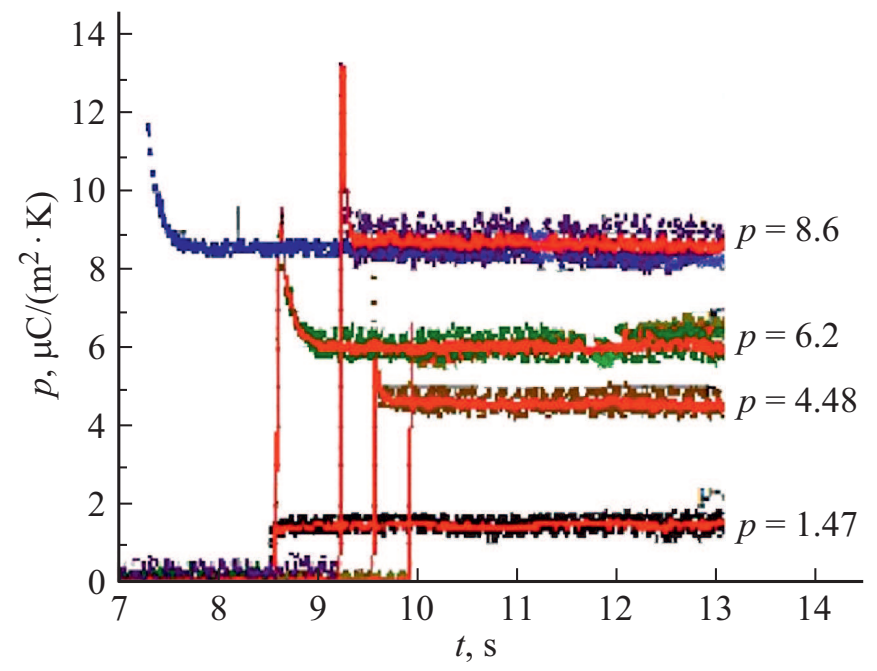

Рис. 1. Постимпульсная релаксация пирокоэффициента $p(t)$ для четырех образцов со структурой типа „AlN на $\mathrm{Si}^{\circ}$ c различной толщиной $\mathrm{AlN}$-слоя. Справа представлены значения пирокоэффициента в стационарном режиме.

Значения пирокоэффициентов $p_{j}$ для различных значений $W_{j}$ толщины AlN-слоя

\begin{tabular}{c|c}
\hline$W_{j}, \mu \mathrm{m}$ & $p_{j}, \mu \mathrm{C} /\left(\mathrm{m}^{2} \cdot \mathrm{K}\right)$ \\
\hline 18 & 1.47 \\
40 & 1.62 \\
48 & 2.5 \\
80 & 3.8 \\
84 & 4.48 \\
89 & 5.54 \\
110 & 6.2
\end{tabular}

дала с площадью подложки и электродов и составляла $1.0-1.5 \mathrm{~cm}^{2}$. Толщина AlN-слоя для различных образцов находилась в пределах $10-170 \mu \mathrm{m}$, а толщина Si-подложки была для всех образцов одинаковой и составляла $400 \mu \mathrm{m}$.

Методика определения значений пирокоэффициента состояла в одновременном измерении пиротока и независимом бесконтактном измерении самой температуры и ее изменений в рабочем слое, происходящих в результате теплового воздействия импульсным лазерным излучением. Для этой цели был задействован лазерный модуль STLL MM-635-120-S3 на основе полупроводникового лазера с длиной волны $\lambda_{l}=0.98 \mu \mathrm{m}$ и регулируемой мощностью излучения (до $120 \mathrm{~mW}$ ). Модулированное излучение лазера обеспечивало динамическое тепловое воздействие на контактный электрод лицевой поверхности AlN-слоя в форме ступенчатой функции [9].

Динамика изменения температуры поверхности образца регистрировалась с помощью неохлаждаемых пироприемников, собранных на базе фотодиодов среднего ИК-диапазона $\left(\Delta \lambda_{s}=3.4-4.4 \mu \mathrm{m}\right.$, www.ioffeled.com).
Точность регистрации была не хуже $0.05^{\circ} \mathrm{C}$. Важным достоинством использованных фотодиодов являлось полное отсутствие чувствительности к излучению на длине волны генерации лазера. Измерения проводились по методике, предложенной нами ранее [11], позволяющей учитывать наличие теплообмена исследуемого образца с окружающей воздушной средой. Определение пирокоэффициента $p(t)$ производилось согласно соотношению

$$
p=i_{p y r}\left(\frac{d T}{d t}\right)^{-1}=U_{p y r}\left[K_{p y r}\left(\frac{d T}{d t}\right)\right]^{-1},
$$

где $i_{p y r}-$ пироэлектрический ток, $T-$ температура поверхности образца, $U_{p y r}$ - регистрируемое напряжение пироэлектрического сигнала, $K_{p y r}-$ коэффициент преобразования ток-напряжение в электронной схеме детектирования пироэлектрического тока.

В соответствии с выражением (1) совместное постимпульсное измерение релаксации температуры поверхности структуры и релаксации пироэлектрического сигнала $U_{p y r}(t)$ позволяет провести вычисление текущих значений пирокоэффициента $p(t)$. Результаты подобных вычислений для некоторых из исследованных AlN/Si-образцов, различающихся толщиной $W$ рабочего слоя AlN, представлены на рис. 1. Значения пирокоэффициентов, соответствующие квазистационарным условиям [9], для полного набора AlN/Si-структур приведены в таблице.

Наблюдаемая зависимость пирокоэффициента от толщины $W_{j}$, очевидно, является следствием неоднородности $z$-компоненты (c-направление) поляризации $P_{z}(z)$, монотонно возрастающей по мере удаления от гетерограницы AlN/Si, что может быть вызвано, в частности, наличием упругих напряжений. Основной причиной возникновения этих напряжений является, как известно, существенное различие коэффициентов теплового расширения $\left(\alpha_{\mathrm{Si}}=3.72 \cdot 10^{-6} \mathrm{~K}^{-1}\right.$ и $\left.\alpha_{11 \mathrm{AlN}}=5.4 \cdot 10^{-6} \mathrm{~K}^{-1}[12,13]\right)$, что в ходе постэпитаксиального остывания структуры приводит к появлению в AlN-слое неоднородных растягивающих деформаций. С целью прояснения этого предположения некоторые из образцов, имеющие достаточную толщину, были подвергнуты травлению в растворе НF до полного удаления Si-подложки и для таких свободных от упругих напряжений квазиобъемных AlN-образцов были выполнены необходимые повторные измерения. В результате этой процедуры величина пирокоэффициента возрастала, причем для образца с $W=110 \mu \mathrm{m}$ она увеличилась более чем на $25 \%$ и достигла $8.6 \mu \mathrm{C} /\left(\mathrm{m}^{2} \cdot \mathrm{K}\right)$. Такое поведение зависимости пирокоэффициента $p(W)$ свидетельствует о возможном его насыщении при $W>300 \mu \mathrm{m}$ вблизи $\sim 9-10 \mu \mathrm{C} /\left(\mathrm{m}^{2} \cdot \mathrm{K}\right)$, что соответствовало бы значению пирокоэффициента объемного материала, полученного, однако, эпитаксиальным методом. Эта зависимость представлена на рис. 2, где для сравнения приведена аналогичная зависимость для $\mathrm{AlN} / \mathrm{SiC}$-структуры. 


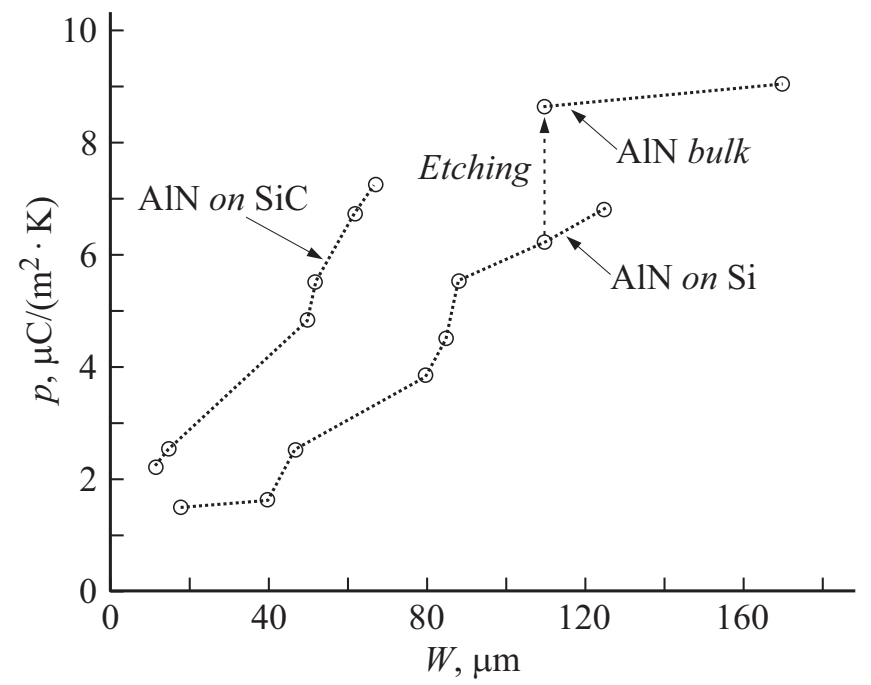

Рис. 2. Зависимость пироэлектрического коэффициента от толщины слоя AlN для AlN/Si-cтруктуры и для AlN-слоя после удаления подложки (AlN bulk). Для сравнения приведена аналогичная зависимость для AlN/SiC-структуры.

Сравнение этих кривых показывает, что в случае $\mathrm{SiC}-$ подложки значения пирокоэффициента по мере возрастания ее толщины быстрее стремятся к насыщению, которое соответствует недеформированному объемному материалу, что может быть связано не только с различием в характере упругих полей. Другими возможными причинами меньших пирокоэффициентов в случае эпитаксии на кремнии являются, во-первых, высокая концентрация дислокаций несоответствия, обусловленная значительным рассогласованием в параметрах решетки (для кремния $a_{\mathrm{Si}}=0.543 \mathrm{~nm}$, для $\mathrm{AlN} a_{\mathrm{AlN}}=0.311 \mathrm{~nm}$ ), и, во-вторых, более интенсивная диффузия атомов $\mathrm{Si}$ в растущий AlN-слой. По всей видимости, оба эти фактора приводят к увеличению вблизи подложки концентрации различных точечных дефектов и их комплексов, способствующих в свою очередь разориентации внутренних дипольных моментов и локальному снижению $c$-компоненты вектора спонтанной поляризации.

Тем не менее сопоставление полученных результатов с теми немногочисленными данными, которые были представлены другими исследовательскими группами, использующими иные AlN-технологии, показывает, что величина пирокоэффициента, зафиксированная в нашем случае для „толстых“ слоев на подложке $\left(p \sim 7 \mu \mathrm{C} /\left(\mathrm{m}^{2} \cdot \mathrm{K}\right)\right)$ и квазиобъемного материала $\left(p=8.6-9.0 \mu \mathrm{C} /\left(\mathrm{m}^{2} \cdot \mathrm{K}\right)\right)$, сопоставима с аналогичными значениями как для объемных пластин $\left(p \sim 5 \mu \mathrm{C} /\left(\mathrm{m}^{2} \cdot \mathrm{K}\right)\right) \quad[14]$, так и для эпитаксиальных субмикронных AlN-пленок, полученных на кремнии MOCVD-методом $\left(p=6-8 \mu \mathrm{C} /\left(\mathrm{m}^{2} \cdot \mathrm{K}\right)\right)[15]$ и пленок толщиной $\sim 1 \mu \mathrm{m}$, полученных методом магнетронного распыления $\left(p=10-12.4 \mu \mathrm{C} /\left(\mathrm{m}^{2} \cdot \mathrm{K}\right)\right)[16]$.

Необходимо учитывать, однако, что конкретные технологии ориентированы на определенный круг при- ложений, и в отличие от технологий для объемного материала эпитаксиальные технологии открывают пути для интегрирования $\mathrm{AlN}$-элементов с кремниевыми микросхемами, которые могли бы обеспечить, например, первичную обработку сигналов. Так, в частности, тонкие AlN-пленки могут быть использованы в микросхеме в основном для создания матриц пирометрических сенсоров. В то же время возможность получения слоев в широком диапазоне их толщин, обеспечиваемая высокой скоростью роста при HVPE-процессе, открывает в свою очередь дополнительные пути повышения уровня однокристального интегрирования, включающего, в частности, микромеханические пьезоэлементы, при сохранении всех возможностей для реализации пироэлектрической микросенсорики.

\section{Конфликт интересов}

Авторы заявляют, что у них нет конфликта интересов.

\section{Список литературы}

[1] Semond F., Lorenzini P., Grandjean N., Massies J. // Appl. Phys. Lett. 2001. V. 78. N 3. P. 335-337.

[2] Raghavan S., Redwing J.M. // J. Appl. Phys. 2004. V. 96. N 5. P. 2995-3003.

[3] Sharofidinov Sh.Sh., Golovatenko A.A., Nikitina I.P., Seredova N.V., Mynbaeva M.G., Bougrov V.E., Odnobludov M.A., Stepanov S.I., Nikolaev V.I. // Mater. Phys. Mech. 2015. V. 22. N 1. P. 53-58.

[4] Шарофидинов Ш.Ш., Кукушкин С.А., Редьков А.В., Гращенко А.С., Осипов А.В. // Письма в ЖТФ. 2019. Т. 45. B. 14. C. 24-27.

[5] Fraga M.A., Furlan H., Pessoa R.S., Massi M. // Microsyst. Technol. 2014. V. 20. N 1. P. 9-21.

[6] Kim T., Kim J., Dalmau R., Schlesser R., Preble E., Jiang X. // IEEE Trans. Ultrason. Ferroelectr. Frequency Control. 2015. V. 62. N 10. P. 1880-1887.

[7] Yan W.S., Zhang R., Xiu X.Q., Xie Z.L., Han P., Jiang R.L., Gu S.L., Shi Y., Zheng Y.D. // Appl. Phys. Lett. 2007. V. 90. N 21. P. 212102 (1-3).

[8] Bukhovski A.D., Kaminski V.V., Shur M.S., Chen Q.C., Khan M.A. // Appl. Phys. Lett. 1996. V. 69. N 21. P. 3254 3256.

[9] Гаврилов Г.А., Капралов А.Ф., Муратиков К.Л., Панютин Е.А., Сотников А.В., Сотникова Г.Ю., Шаробидинов Ш.Ш. // Письма в ЖТФ. 2018. Т. 44. В. 16. С. 11-19.

[10] Кукушкин С.А., Осипов А.В., Сергеева О.Н., Киселев Д.А., Богомолов А.А., Солнышкин А.В., Каптелов Е.Ю., Сенкевич С.В., Пронин И.П. // ФТТ. 2016. Т. 58. В. 5. С. 937-940.

[11] Александров С.Е., Гаврилов Г.А., Капралов А.А., Муратиков К.Л., Сотникова Г.Ю. // Письма в ЖТФ. 2017. Т. 43. B. 23. C. $77-85$.

[12] Figge S., Krïce H., Hommel D., Epelbaum E.M. // Appl. Phys. Lett. 2009. V. 94. N 10. P. 101915. 
[13] Ivanaga H., Kunishige A., Takeuchi S. // J. Mater. Sci. 2000. V. 35. N 10. P. 2451-2454.

[14] Шалдин Ю.B., Matyjasik S. // ФТП. 2011. Т. 45. В. 9. C. 1159-1165.

[15] Fuflyigin V., Salley E., Osinsky A., Norris P. // Appl. Phys. Lett. 2000. V. 77. N 19. P. 3075-3077.

[16] Stan G.E., Botea M., Boni G.A., Pintilie I., Pintilie L. // Appl. Surf. Sci. 2015. V. 353. P. 1195-1202. 